\title{
Optimal Wind-Thermal Coordination Scheduling Considering Reserve Requirement
}

\author{
Chan-Mook Jung \\ Department of Railroad and Civil Engineering, \\ Woosong University, Daejeon - 34606, South Korea. \\ E-mail: cmjung@wsu.ac.kr \\ Salkuti Surender Reddy \\ Department of Railroad and Electrical Engineering, \\ Woosong University, Daejeon - 34606, South Korea. \\ Corresponding author: surender@wsu.ac.kr
}

(Received March 27, 2019; Accepted August 29, 2019)

\begin{abstract}
This paper proposes an optimal coordination scheduling methodology for a wind-thermal power system by considering the intermittent behavior of wind power and load demands. The optimum amount of spinning reserves (SRs) needed to handle the uncertain nature of hybrid power system with wind generators is determined by using the probability analysis. The proposed model demonstrates the error in wind power and load forecasts by using the probability distribution functions for over and under estimations of load and wind power. In this work, two objectives, i.e., total operating cost and risk level are considered, which handles both economical and reliability issues, simultaneously. This problem is solved by using the meta-heuristic based Cuckoo search algorithm. The reactive power capability limits of synchronous generators and variable speed doubly fed induction generators are considered in this paper. Simulations have been carried out on IEEE 300 bus system.
\end{abstract}

Keywords- Renewable energy sources, Spinning reserves, Uncertainty, Optimal scheduling, Evolutionary algorithms.

\section{Introduction}

Nowadays, wind energy has drawn much attention compared to other renewable sources of energy. Increased development and deployment of green/renewable energy technologies rank amongst the top priorities of many countries. The search for the development and utilization of RERs has coincidentally, aligned the interests of both industrialized and developing nations. The industrialized countries need to satisfy increases in power demand without increasing their dependence on foreign importation of oil and extra degradation of the environment. The developing countries see renewable energy as a way of meeting increasing power demands and extending electricity usage to disperse population with reduced stress on national electric network and also to take benefit of carbon credit. This alignment of interests will spur economic growth because any successful technology developed in one part of the world can easily be deployed in any other part of the world. This will also require the developments of standards and quality that is acceptable to the international community. RERs such as wind and solar power generations are uncertain in nature and new energy storage technologies are required to overcome the difficulties posed by these characteristics. Increasing concerns about energy security, climate change, and diminishing the reserves of fossil fuels around the world are leading the increased interest in generation, distribution, and management of renewable power. However, the intermittent nature of RERs in a challenging problem to address. 
International Journal of Mathematical, Engineering and Management Sciences

Vol. 5, No. 1, 108-119, 2020

https://doi.org/10.33889/IJMEMS.2020.5.1.010

Raising contribution from renewable energy resources (RERs) will have several benefits such as reduced air pollution, global climate and economic benefit, etc. Therefore, it is advantageous to use renewable energy resources to the existing conventional thermal power generation. However, the major drawback is they are intermittent and unpredictable in nature. Wind power prediction applications are one of the tools that have been recently developed to help power system operation by estimating the future wind farm power production (Jabr and Pal, 2009). For this, meteorological forecasts are used to collect the data from wind farm. Prediction will help in power system operation by estimating the future wind power and to anticipate congestion in the system, power reserve requirement or prevent any adverse effects in under/over voltage. The aim of wind power prediction programs is to make wind energy generators to participate actively in the electricity markets, in spite of its non-dispatch able/non-schedulable nature (Liu and Xu, 2010).

In order to evaluate reactive power ancillary service requirement in the wind plant, it is advantageous to determine the capability curve of wind turbines and wind variation into the account. Recently, the variable speed wind turbine attracted many people because of its increased energy production. The advantage of using variable speed doubly fed induction generator (DFIG) is its capacity to transfer maximum power in sub and super-synchronous speeds. The optimal scheduling problem including thermal units and wind generators is a combination of load flow and economic dispatch problems. The objective of this problem can be minimization of fuel costs, power loss, reactive power loss, load shedding and also maximize voltage security index, branch flow security index, etc. The objective function is subjected to various constraints, and due to this the optimal scheduling problem becomes a non-linear mathematical programming problem (Wood et al., 2013). A classical economic dispatch (ED) model by including the wind energy conversion system and factors to account for both under and over estimations of wind power has been presented in Hetzer et al. (2008).

Spinning reserve (SR) is a type of ancillary service, and it helps to maintain the reliability of system in case of unforeseen and sudden events like line/generator outages, demand changes or both (Rebours and Kirschen, 2005). Damodaran and Kumar (2018) proposed a wind-hydrothermal scheduling problem with economic emission factors in a complex non-linear multiobjective optimization point of view. A risk-based SR allocation approach by considering multiple control sub-area coordination is proposed in reference Chen et al. (2013). Zhou et al. (2011) proposed a dynamic economic dispatch (DED) with wind power by considering uncertainties due to wind speed, load forecasts and generation forced outages. A day-ahead DED approach for the power system with wind energy generators (WEGs) to optimize cost of generation and reserves of all generating units is presented in Chao et al. (2015). Singh et al. (2009) proposed 4 versions of particle swarm optimization to solve the scheduling problem of wind-thermal power system. A simulated annealing method combined with an efficient constrained DED approach is presented in Chen (2007) to coordinate thermal and wind generation coordination in a power system with large scale penetration of wind power.

This paper integrates the wind energy generating systems in optimal scheduling model by handling both economical and reliability issues, simultaneously. Different factors involved in the intermittency of wind power and load are analyzed. The variations of wind power and load forecasting errors in under and over-estimation of the power production are presented in this paper. Two important and conflicting objectives, i.e., total operating cost and risk level are optimized in this paper in the presence of wind and load demand forecast uncertainties. This 
International Journal of Mathematical, Engineering and Management Sciences

Vol. 5, No. 1, 108-119, 2020

https://doi.org/10.33889/IJMEMS.2020.5.1.010

paper also characterizes the uncertain nature of wind speed and wind power. Probability distribution function (PDF) of WEG's power output in both discrete and continuous portions is presented. The SRs requirement and the excess power that the wind farms and loads that might produce from the shape and scale factor of Weibull distribution, and Normal distribution functions. The reactive power capabilities of synchronous generator and doubly fed induction generators (DFIGs) are included in the proposed optimization model. Simulations have been carried out on standard IEEE 300 bus system.

The present paper is organized as follows. Section 2 presents the problem formulation of optimal wind-thermal scheduling. Modeling of wind speed, power and load demand is presented in Section 3. The detailed description of Cuckoo Search Algorithm (CSA) is presented in Section 4. Section 5 presents results and discussion on IEEE 300 bus system. Finally, conclusions are reported in Section 6.

\section{Optimal Wind-Thermal Scheduling: Problem Formulation}

Renewable energy has been given more and more importance due to its energy saving and environmentally friendly nature. However, due to unpredictability and intermittency nature of RERs, many problems will arise when wind power is integrated into the network with conventional thermal generators and finds difficulty in dispatching this power. It is well known that electricity cannot be easily stored, and this explains that fluctuations in wind power must be handled by other types of generation. This uncertainty emphasizes the system operation and may involve additional cost to the electricity due to power imbalance. Therefore, every participant in the electricity market will be interested in reducing this imbalance cost as much as possible. Aiming at these difficulties, a reasonable trade-off between the risk and total operating cost is desired. Therefore, it is clearly understood that optimal scheduling incorporating the wind power should also take care of factors involved in the uncertainty in addition to operating cost (Laia et al., 2016).

\subsection{Objective 1: Minimization of Total Operating Cost}

The proposed optimal scheduling problem can be considered as a constraint optimization. This objective aims at optimizing the fuel cost and spinning reserves cost of thermal units, wind power cost from private owner, and reducing the risk involved due to wind power and load demand forecast intermittencies. This objective function can be formulated as (Hemmati, 2018),

minimize,

$$
\begin{aligned}
& \sum_{i=1}^{N_{g}}\left[C_{i}\left(P_{g i}\right)+C_{S R i}\left(P_{S R i}\right)\right]+\sum_{j=1}^{N_{w}}\left[C_{w j}\left(P_{w j}\right)+C_{p, w j}\left(P_{w j, a v g}-P_{w j}\right)+C_{r, w j}\left(P_{w j}-\right.\right. \\
& \left.\left.P_{w j, a v g}\right)\right]+\sum_{k=1}^{N_{D}}\left[C_{p, D k}\left(P_{D k, a v g}-P_{D k}\right)+C_{r, D k}\left(P_{D k}-P_{D k, a v g}\right)\right]
\end{aligned}
$$

First term in equation (1) is cost of thermal units, and it is expressed as (Hetzer et al., 2008),

$$
C_{i}\left(P_{g i}\right)=a_{i} P_{g i}^{2}+b_{i} P_{g i}+c_{i}
$$

where $a_{i}, b_{i}, c_{i}$ are the fuel cost coefficients of $i^{\text {th }}$ conventional unit. Second term is SRs cost of thermal generators, and it is expressed as (Reddy et al., 2015),

$$
C_{S R i}\left(P_{S R i}\right)=x_{i}+y_{i} P_{S R i}
$$


International Journal of Mathematical, Engineering and Management Sciences

Vol. 5, No. 1, 108-119, 2020

https://doi.org/10.33889/IJMEMS.2020.5.1.010

Third term represents the operating cost for power drawn from wind energy generator and it is expressed as (Jabr and Pal, 2009),

$C_{w j}\left(P_{w j}\right)=d_{j} P_{w j}$

Fourth term is cost associated with not using all the available wind power (under-estimation), and this cost is termed as penalty cost. The uncertain nature of wind power is a major barrier to its integration into utility grid. Therefore, to account this variation two cost function model is proposed. This cost function helps us to find excess power it might produce that the scheduled power (Ji et al., 2018). Mathematically, this penalty cost function is expressed as,

$C_{p, w j}\left(P_{w j, a v g}-P_{w j}\right)=K_{P, j}\left(P_{w j, a v g}-P_{w j}\right)=K_{p, j} \int_{P_{w j}}^{P_{r, j}}\left(p-P_{w j}\right) f_{p}(p) d w$

Fifth term represents the cost that must be paid for over-estimation of available wind power. This cost accounts the possibility of reserve need to be drawn. The cost function can be expressed as,

$C_{r, w j}\left(P_{w j}-P_{w j, a v g}\right)=K_{r, j}\left(P_{w j}-P_{w j, a v g}\right)=K_{r, j} \int_{0}^{P_{w j}}\left(P_{w j}-p\right) f_{p}(p) d w$

Sixth and seventh terms depicts under-estimation (i.e., penalty cost) and over-estimation (i.e., reserve cost) of load demand, respectively, and they are expressed as,

$$
\begin{aligned}
& C_{p, D k}\left(P_{D k, a v g}-P_{D k}\right)=K_{P, k}\left(P_{D k, a v g}-P_{D k}\right)=K_{p, k} \int_{P_{D k}}^{P_{r, k}}\left(p-P_{D k}\right) f_{l}(p) d l \\
& C_{r, D k}\left(P_{D k}-P_{D k, a v g}\right)=K_{r, k}\left(P_{D k}-P_{D k, a v g}\right)=K_{r, k} \int_{0}^{P_{D k}}\left(P_{D k}-p\right) f_{l}(p) d l
\end{aligned}
$$

\subsection{Objective 2: System Risk Level (R) Minimization}

There is always a risk factor is associated with the penetration of wind power into the existing power system (Ren and Jihong, 2014). In this paper, fuzzy approach is used to represent wind power penetration into the existing power system. A linear fuzzy membership function for wind power penetration $(\mu)$ is used to depict the system security level. This system security level is expressed as (Reddy et al., 2015),

$$
\mu= \begin{cases}1 & \text { if } P_{w} \leq w\left(P_{D}\right)^{\text {min }} \\ \frac{w\left(P_{D}\right)^{\max }-P_{w}}{w\left(P_{D}\right)^{\text {max }}-w\left(P_{D}\right)^{\text {min }}} & \text { if } w\left(P_{D}\right)^{\text {min }}<P_{w}<w\left(P_{D}\right)^{\text {max }} \\ 0 & \text { if } P_{w} \geq w\left(P_{D}\right)^{\text {max }}\end{cases}
$$

Wind power $\left(P_{w}\right)$ defines the fuzzy order. $w\left(P_{D}\right)^{\min }$ and $w\left(P_{D}\right)^{\max }$ are lower and upper bounds 
International Journal of Mathematical, Engineering and Management Sciences

Vol. 5, No. 1, 108-119, 2020

https://doi.org/10.33889/IJMEMS.2020.5.1.010

of wind power penetrations. From equation (9), it can be observed that the system is more secured if the system has larger value of security level $(\mu)$. Therefore, the minimization of system risk is selected as an objective function, and it can be expressed as (Reddy et al., 2015):

$R(\mu)=\frac{1}{\mu}$

\subsection{Equality Constraints}

The equality constraints of proposed optimal scheduling problem contemplate the law of physics in power system, and they are expressed as,

$$
\begin{aligned}
& P_{k}=0=V_{k} \sum_{m=1}^{N_{b}}\left[V_{m}\left[G_{k m} \cos \left(\delta_{k}-\delta_{m}\right)+B_{k m} \sin \left(\delta_{k}-\delta_{m}\right)\right]\right]-P_{G k}-P_{L k} \\
& Q_{k}=0=V_{k} \sum_{m=1}^{N_{b}}\left[V_{m}\left[G_{k m} \sin \left(\delta_{k}-\delta_{m}\right)-B_{k m} \cos \left(\delta_{k}-\delta_{m}\right)\right]\right]-Q_{G k}-Q_{L k}
\end{aligned}
$$

\subsection{Inequality Constraints}

(a) Generator Constraints

Each generator has maximum and minimum power outputs, and they are expressed as ( $\mathrm{Li}$ and Zhao, 2013),

$$
\begin{aligned}
& P_{g i}^{\min } \leq P_{g i} \leq P_{g i}^{\max } \\
& P_{w j} \leq P_{w j}^{\text {max }} \\
& V_{i}^{\text {min }} \leq V_{i} \leq V_{i}^{\text {max }}
\end{aligned}
$$

In addition to the above constrains, the transmission lines have MVA rating so as a part of security of the system, the power flow in a line should not exceeds the maximum rating of transmission lines.

\section{(b) Reactive Power Capability Limits}

Reactive power capability of synchronous generator is constrained by armature and field currents. The armature current results in an $\mathrm{RI}^{2}$ power loss. Hence, MVA rating is given by (Banerjee et al., 2016),

$$
P^{2}+Q^{2} \leq V_{t} I_{a}
$$

Field heating limit is due to the heat resulting from the field circuit; hence the field current imposes a second limit on operation of generator. It is given by, 
International Journal of Mathematical, Engineering and Management Sciences

Vol. 5, No. 1, 108-119, 2020

https://doi.org/10.33889/IJMEMS.2020.5.1.010

$P^{2}+\left(Q+\frac{V_{t}^{2}}{X_{s}}\right)^{2} \leq\left(\frac{V_{t} E_{a f}}{X_{s}}\right)^{2}$

The reactive power generation of wind generator (i.e., doubly fed induction generator (DFIG)) can be controlled by rotor current, and it is expressed as,

$$
\begin{aligned}
& Q_{s} \geq-\frac{v_{t}^{2}}{\omega_{s}\left(L_{s}+L_{m}\right)}-\frac{L_{m} v_{t}}{\left(L_{s}+L_{m}\right)} \sqrt{I_{r}^{\max ^{2}}-\left[\frac{P_{s}\left(L_{s}+L_{m}\right)}{v_{t} L_{m}}\right]^{2}} \\
& Q_{s} \leq-\frac{v_{t}^{2}}{\omega_{s}\left(L_{s}+L_{m}\right)}+\frac{L_{m} v_{t}}{\left(L_{s}+L_{m}\right)} \sqrt{I_{r}^{\max ^{2}}-\left[\frac{P_{s}\left(L_{s}+L_{m}\right)}{v_{t} L_{m}}\right]^{2}}
\end{aligned}
$$

\section{(c) Spinning Reserve (SR) Requirement Constraints}

Here, the SR requirement is determined by protecting the power system against the contingency of largest online conventional thermal generator, and uncertainties due to wind power and load demand forecasts. Hence, total amount of spinning reserves required $\left(T S R_{r e q}\right)$ is expressed as (Reddy et al., 2015),

$T S R_{\text {req }}=P_{G, \text { largest }}+\sum_{j=1}^{N_{W}}\left|P_{w j}-P_{w j, a v}\right|+\sum_{k=1}^{N_{D}}\left|P_{D k}-P_{D k, \text { avg }}\right|$

\section{Modeling of Wind Speed and Power}

Wind prediction method based on fuzzy logic, neural network (NN), artificial intelligence etc has some unique features and certain degree of accuracy. Statistical data are not stationary and there are weak diurnal variation and change trend suddenly they may lead to very inaccurate results. All current prediction programs use at least one of the two kinds of methods of prediction wind power, they are: physical and statistical methods. Recent research shows combination of both physical and statistical method will provide a diagnostic value. In this paper, Weibull PDF is used as it is simple and provided a good fit to procured wind speed data.

\subsection{Characterization of Wind Speed}

To determine power output from wind energy generator (WEG), it is required to know the wind speed profile at a particular location. Here, scale (c) and shape (k) parameters are used to depict the variation in wind speed. These parameters depend upon height, location and some geographical features. Wind speed is modeled by using the Weibull PDF, and it is described as (Han et al., 2015),

$f_{v}(v)=\left(\frac{k}{c}\right) *\left(\frac{v}{c}\right)^{(k-1)} \exp \left[-\left(\frac{v}{c}\right)^{k}\right] \quad 0<v<\infty$

From scale and shape parameters, one can easily estimate the mean and standard deviation of distribution curve, and they are given by, 


$$
\begin{aligned}
& E(V)=c * \Gamma\left(1+k^{-1}\right)=\frac{c}{k} * \Gamma\left(\frac{1}{k}\right) \\
& \operatorname{var}(V)=E\left(V^{2}\right)-E^{2}(V)=\frac{c^{2}}{k}\left[2 \Gamma\left(\frac{2}{k}\right)-\frac{1}{k}\left[\Gamma\left(\frac{1}{k}\right)^{2}\right]\right]
\end{aligned}
$$

The power output from WEG for a given wind speed input $(v)$ can be expressed as,

$$
p=\left\{\begin{array}{cc}
0 & \text { for } v<v_{i} \text { and } v>v_{o} \\
p_{r} * \frac{\left(v-v_{i}\right)}{\left(v_{r}-v_{i}\right)} & \text { for } v_{i} \leq v \leq v_{r} \\
p_{r} & \text { for } v_{r} \leq v \leq v_{o}
\end{array}\right.
$$

\subsection{Wind Power Output in Continuous Range}

From equation (24), it can be observed that there is a linear increase in the wind power in the region between cut-in to rated wind speeds. Hence, it is a continuous distribution in this range. The PDF in continuous range can be expressed by (Zhang et al., 2018).

$$
f_{p}(p)=\frac{k\left(v_{r}-v_{i}\right)}{c^{k} * p_{r}}\left[v_{i}+\frac{p}{p_{r}}\left(v_{r}-v_{i}\right)^{k-1}\right] \exp \left[-\left[\frac{v_{i}+\frac{p}{p_{r}}\left(v_{r}-v_{i}\right)}{c}\right]^{k}\right]
$$

\subsection{Wind Power Output in Discrete Range}

From equation (24), it can also be observed that there are two discrete probabilities occurs, and the probability of event when there is no wind power output $(\mathrm{P}=0)$ is given by,

$$
P_{r}(P=0)=1-e\left(-\left(\frac{v_{i}}{c}\right)^{k}\right)+e\left(-\left(\frac{v_{o}}{c}\right)^{k}\right)
$$

Probability of event at rated power output $\left(\mathrm{P}=\mathrm{P}_{\mathrm{r}}\right)$ is given by,

$$
P_{r}\left(P=P_{r}\right)=e\left(-\left(\frac{v_{r}}{c}\right)^{k}\right)+e\left(-\left(\frac{v_{o}}{c}\right)^{k}\right)
$$

From equations (25) -(27), it can be observed that it is mixed probability distribution function.

\subsection{Calculation of Reserve Requirement}

The amount of active power reserve required only if the actual wind power is less than the scheduled one. Hence, the system operator (SO) requires to buy power from another available power source. In equation (1), for the $\mathrm{j}^{\text {th }}$ wind energy generator, cost of over-estimation is expressed as $C_{r, w j}\left(P_{w j}-P_{w j, a v g}\right)$, where $C_{r, w j}$ is cost coefficient for purchasing power from other available power sources. Let scheduled wind power for a particular wind turbine is denoted by $P_{r}\left(0 \leq P_{w j}<P_{r}\right)$, then the reserve requirement can be expressed by $X_{o}=\left(P_{w j}-P\right)$, and it is described as, 
International Journal of Mathematical, Engineering and Management Sciences

Vol. 5, No. 1, 108-119, 2020

https://doi.org/10.33889/IJMEMS.2020.5.1.010

$X_{o}=P_{w j}\left[\left.F_{p}\left(P_{w j}\right)\right|_{P_{w j \neq 0}}+F_{p}(0)\right]-\int_{0}^{P_{w j}} p f_{p}(p) d p$

From the above equation, it can be observed that $X_{O}$ depends upon the scale and shape factors, and $X_{o}$ is nonlinear in terms of $P_{w j}$.

\subsection{Calculation of Excess Power}

There might be a chance that the produced wind power more than the expected value. The excess power produced can be expressed by $X_{u}=\left(P-P_{w j}\right)$, and it is described as,

$$
X_{u}=\int_{P_{w j}}^{P_{r}} p f_{p}(p) d p-P_{w j} \int_{P_{w j}}^{P_{r}} f_{p}(p) d p
$$

This equation also depends upon scale and shape factors, and $X_{u}$ is nonlinear in terms of $P_{w j}$.

\section{Cuckoo Search Algorithm (CSA)}

As explained earlier, this paper considers both over and under-estimations of wind power and load demands along with classical economic factors of thermal and wind energy generators. The classical optimization techniques can be used by including the WEGs in the optimal scheduling problem. However, the difficulty is finding the derivatives with respect to objective cost components (i.e., over and under estimation components) are not easily found (Reddy, 2017). Therefore, the stochastic model of wind power in the optimal scheduling problem is solved by using the evolutionary based CSA. It is an evolutionary based optimization algorithm which is nature-inspired by Cuckoos' search for their nests where they could lay their eggs (Jiang et al., 2016). For detailed analysis of CSA, the reader may refer reference Rajabioun (2011). The flow chart of CSA is presented in Figure 1.

\section{Results and Discussion}

In this work, simulation studies have been performed on IEEE 300 bus system, which consists of 69 generators, among them 57 are thermal generating units and 12 are considered as the wind farms. The maximum capacity of each wind plant is selected as 250MW. All optimization programs are coded in MATLAB R2018b and executed on a personal computer with Intel i7, 8 GB RAM and $3.1 \mathrm{GHz}$ processor. A MATLAB program based model is developed to provide a numerical tool to solve the proposed problem. This stochastic model is integrated in Newton Raphson load flow in which the wind energy generators are represented by variable speed DFIGs. Here, three case studies are performed on IEEE 300 bus system, and they are:

- Case Study 1: Total operating cost (TOC) minimization with no uncertainties in wind power and load forecasts.

- Case Study 2: TOC minimization with uncertainty only in wind power and no uncertainty in load forecast.

- Case Study 3: TOC minimization with uncertainties in both wind power and load forecasts. 
International Journal of Mathematical, Engineering and Management Sciences

Vol. 5, No. 1, 108-119, 2020

https://doi.org/10.33889/IJMEMS.2020.5.1.010

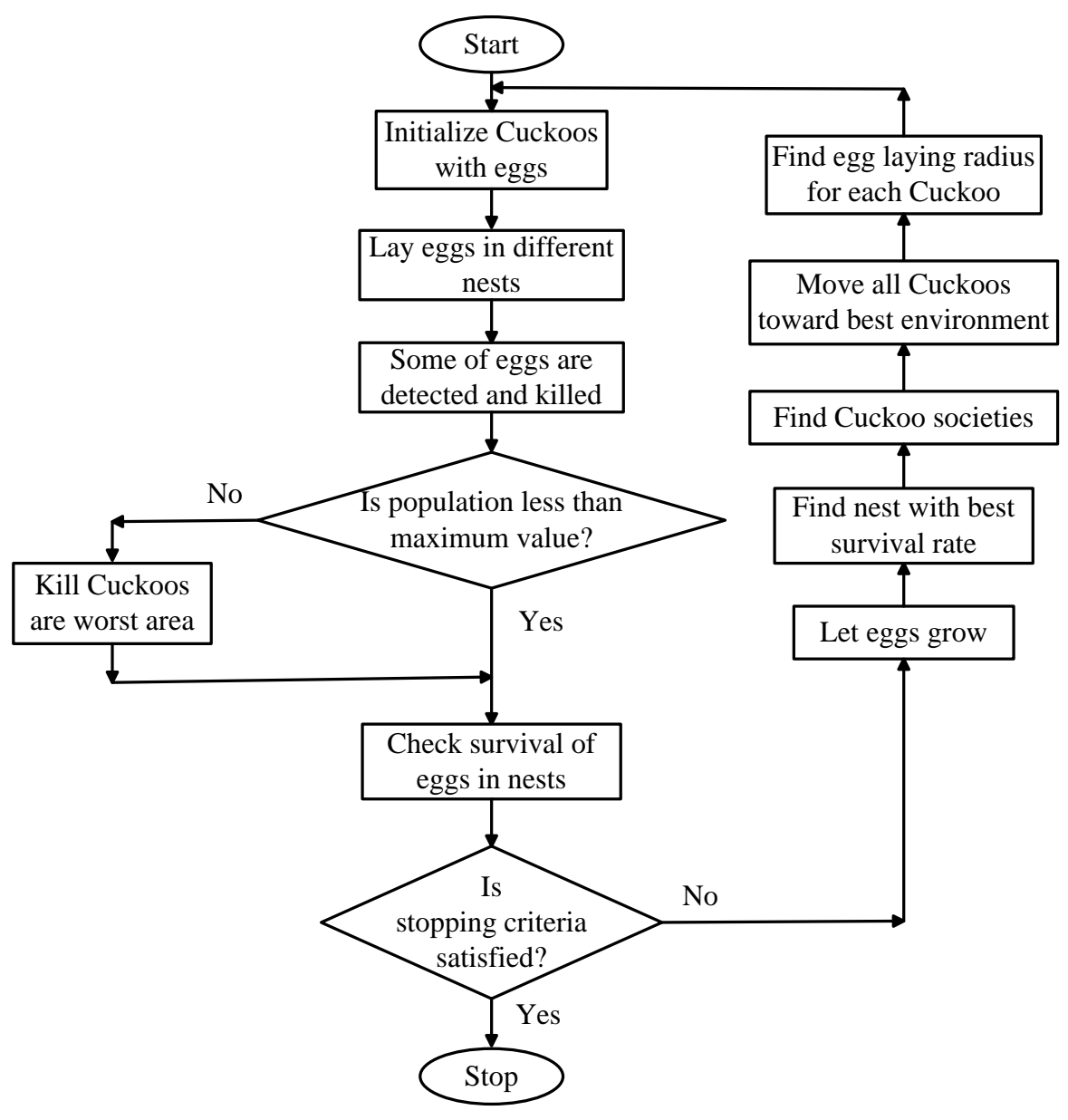

Figure 1. Flow chart of Cuckoo search algorithm

\subsection{Case Study 1}

Table 1 presents the objective function values for this Case study 1 . As stated earlier, this case doesn't consider any uncertainties in wind power and load forecasts. In this case, minimization of total operating cost objective includes only energy cost, spinning reserves (SRs) cost of conventional generators, and energy cost of WEGs. Here, the total amount of spinning reserves needed is equal to the scheduled power of largest online thermal generator, which is 1906.5201MW. The obtained optimal total operating cost is $953572.2064 \$ / \mathrm{hr}$, which is the sum of thermal power cost (796873.3798 \$/hr), wind generation cost (37304.5787 \$/hr), and the SRs cost of thermal generators (119394.2479 \$/hr).

Table 1. Objective function values for Case Study 1

\begin{tabular}{|c|c|}
\hline Cost of thermal and wind power generation $(\$ / \mathrm{hr})$ & 834177.9585 \\
\hline Spinning reserves (SRs) cost of thermal generators $(\$ / \mathrm{hr})$ & 119394.2479 \\
\hline Total operating cost (TOC) $(\$ / \mathbf{h r})$ & $\mathbf{9 5 3 5 7 2 . 2 0 6 4}$ \\
\hline Total amount of SRs required $(\mathbf{M W})$ & $\mathbf{1 9 0 6 . 5 2 0 1}$ \\
\hline
\end{tabular}


International Journal of Mathematical, Engineering and Management Sciences

Vol. 5, No. 1, 108-119, 2020

https://doi.org/10.33889/IJMEMS.2020.5.1.010

\subsection{Case Study 2}

Table 2 presents the objective function values and the amount of SRs needed for Case 2. As explained earlier, the objective function considered in the case study consists of first five terms of Equation (1). As this case considers the uncertainty in wind power, some extra amount of spinning reserves has to be maintained in the existing power system. Here, total amount of SRs needed is the sum of largest online conventional generation (1955.0672 MW), and the amount of SRs needed due to the uncertainty of wind power (529.3892 MW). Hence, the total amount of spinning reserves required in this case is $2484.4564 \mathrm{MW}$. The optimum TOC obtained in this case is $1021233.6705 \$ / h r$, which is the sum of generation cost $(859700.3294 \$ / h r)$, and SRs cost $(158972.2183 \$ / h r)$. Because of the uncertainty in wind power, the TOC obtained in this case is higher than Case 1.

Table 2. Objective function values for Case Study 2

\begin{tabular}{|l|c|}
\hline Cost of thermal and wind power generation $(\$ / \mathrm{hr})$ & 859700.3294 \\
\hline Spinning reserves cost of thermal generators $(\$ / \mathrm{hr})$ & 158972.2183 \\
\hline Total operating cost $\mathbf{\$} / \mathbf{h r})$ & $\mathbf{1 0 2 1 2 3 3 . 6 7 0 5}$ \\
\hline Total amount of SRs required $(\mathbf{M W})$ & $\mathbf{2 4 8 4 . 4 5 6 4}$ \\
\hline System security level $(\mu)$ & 0.2775 \\
\hline System risk level $(\mathbf{R})$ & $\mathbf{3 . 6 0 3 6}$ \\
\hline Amount of reserves required for wind farms $(\mathbf{M W})$ & $\mathbf{5 2 9 . 3 8 9 2}$ \\
\hline Excess power available with wind farms $(\mathbf{M W})$ & 367.6188 \\
\hline
\end{tabular}

As explained earlier, security level $(\mu)$ is determined in terms of wind power penetration by using linear fuzzy model, then risk level $(\mathrm{R})$ is calculated using $\mu$. Here, the security and the risk levels obtained are 0.2775 and 3.6036 , respectively.

\subsection{Case Study 3}

Table 3 presents the objective function value and amount of spinning reserves needed for Case 3 . Here, the objective function (i.e., TOC) consists all the terms of Equation (1). As this case considers uncertainties in both wind power and loads, some extra amount of reserves has to be maintained in the power system. The total amount of spinning reserves needed is the sum of largest online thermal power generation (1955.0672 MW), amount of SRs needed due to the uncertainties in wind power (536.1735 MW), and load uncertainty (84.3391 MW). Hence, the total amount of SRs needed in this case is 2575.5798 MW. In this case, the optimum TOC obtained is $1046782.2470 \$ / h r$, which is the sum of generation cost $(861137.0718 \$ / h r)$, and SRs cost $(185645.1752 \$ / h r)$. Because of the uncertainties in wind power and load demands, the TOC obtained in this case is higher than the Cases 1 and 2. Here, system security and risk levels obtained are 0.2624 and 3.8109 , respectively.

Table 3. Objective function values for Case Study 3

\begin{tabular}{|c|c|}
\hline Cost of thermal and wind power generation $(\$ / \mathrm{hr})$ & 861137.0718 \\
\hline Spinning reserves (SRs) cost of thermal generators (\$/hr) & 185645.1752 \\
\hline Total operating cost $\mathbf{\$} / \mathbf{h r})$ & $\mathbf{1 0 4 6 7 8 2 . 2 4 7 0}$ \\
\hline Total amount of SRs required $(\mathbf{M W})$ & $\mathbf{2 5 7 5 . 5 7 9 8}$ \\
\hline System security level $(\mu)$ & 0.2624 \\
\hline System risk level $(\mathbf{R})$ & $\mathbf{3 . 8 1 0 9}$ \\
\hline Amount of reserves required for wind farms $(\mathbf{M W})$ & 351.6188 \\
\hline Excess power available with wind farms $(\mathrm{MW})$ & $\mathbf{8 4 . 3 3 9 1}$ \\
\hline Amount of reserves required for loads $(\mathbf{M W})$ & 78.2152 \\
\hline Excess power available with loads $(\mathrm{MW})$ & \\
\hline
\end{tabular}


International Journal of Mathematical, Engineering and Management Sciences

Vol. 5, No. 1, 108-119, 2020

https://doi.org/10.33889/IJMEMS.2020.5.1.010

From the case studies presented in this paper, it can be observed that, as the uncertainties in wind power and load demand forecasts increases; total operating cost and risk level of the system increases, which in turn requires additional amount of spinning reserves in the system.

\section{Conclusions}

This paper proposes an approach to integrates wind energy generators in the optimal windthermal scheduling problem by considering wind power and load forecast uncertainties. The stochastic nature of wind speed and power is modeled by Weibull distribution, and load demand is modeled by using Normal distribution. In this work, the factors which accounts for over and under-estimations of wind power and load demands are considered. Here, the spinning reserve (SR) offers from thermal generators is considered. Case studies are executed on IEEE 300 bus test system. The solution of this problem is dependent on different factors like Weibull shape, scale factors, reserve cost for overestimating the wind power, and penalty cost for underestimating the wind power. Simulation results show that cost and reserves have tendency to increase with increase in the level of uncertainty. The obtained optimal solutions also indicate the minimum active and reactive power requirements in wind plant to maintain the satisfactory level of voltages.

\section{Conflict of Interest}

The authors declare that there is no conflict of interest for this publication.

\section{Acknowledgments}

This research work has been carried out based on the support of Woosong University's Academic Research Funding 2019.

\section{References}

Banerjee, S., Dasgupta, K., \& Chanda, C.K. (2016). Short term hydro-wind-thermal scheduling based on particle swarm optimization technique. International Journal of Electrical Power \& Energy Systems, $81,275-288$.

Chao, L., Jun, Y., Zhi, D., Jifeng, H., \& Mingsong, L. (2015). Day-ahead economic dispatch of wind integrated power system considering optimal scheduling of reserve capacity. Energy Procedia, 75, 1044-1051.

Chen, C.L. (2007). Simulated annealing-based optimal wind-thermal coordination scheduling. IET Generation, Transmission \& Distribution, 1(3), 447-455.

Chen, J., Wu, W., Zhang, B., Wang, B., \& Guo, Q. (2013). A spinning reserve allocation method for power generation dispatch accommodating large-scale wind power integration. Energies, 6(10), 5357-5381.

Damodaran, S.K., \& Kumar, T.K.S. (2018). Hydro-thermal-wind generation scheduling considering economic and environmental factors using heuristic algorithms. Energies, 11(2), 1-19.

Han, Z., Cheng, T., Zhou, Y., \& Zhang, P. (2015, November). Multi-objective optimal scheduling for hydro-thermal-wind power system. In TENCON 2015-2015 IEEE Region 10 Conference (pp. 1-5). IEEE. Macao.

Hemmati, R. (2018). Optimal cogeneration and scheduling of hybrid hydro-thermal-wind-solar system incorporating energy storage systems. Journal of Renewable and Sustainable Energy, 10(1), 014102. 
International Journal of Mathematical, Engineering and Management Sciences

Vol. 5, No. 1, 108-119, 2020

https://doi.org/10.33889/IJMEMS.2020.5.1.010

Hetzer, J., Yu, D.C., \& Bhattarai, K. (2008). An economic dispatch model incorporating wind power. IEEE Transactions on Energy Convsion, 23(2), 603-611.

Jabr, R.A., \& Pal, B.C. (2009). Intermittent wind generation in optimal power flow dispatching. IET Generation, Transmission \& Distribution, 3(1), 66-74.

Ji, X., Li, Y., Yu, Y., \& Fan, S. (2018). Optimal dispatching and game analysis of power grid considering demand response and pumped storage. Systems Science \& Control Engineering, 6(3), 270-277.

Jiang, P., Liu, F., Wang, J., \& Song, Y. (2016). Cuckoo search-designated fractal interpolation functions with winner combination for estimating missing values in time series. Applied Mathematical Modelling, 40(23-24), 9692-9718.

Laia, R., Pousinho, H.M., Melício, R., \& Mendes, V.M.F. (2016). Optimal scheduling of joint windthermal systems. In International Joint Conference SOCO'16-CISIS'16-ICEUTE'16 (pp. 136-146). Springer, Cham.

Li, X., \& Zhao, D. (2013). An optimal dynamic generation scheduling for a wind-thermal power system. Energy and Power Engineering, 5(4), 1016-1021.

Liu, X., \& Xu, W. (2010). Minimum emission dispatch constrained by stochastic wind power availability and cost. IEEE Transactions on Power Systems, 25(3), 1705-1713.

Rajabioun, R. (2011). Cuckoo optimization algorithm. Applied Soft Computing, 11(8), 5508-5518.

Rebours, Y., \& Kirschen, D. (2005). A survey of definitions and specifications of reserve services. Report, University of Manchester, 1-38.

Reddy, S.S. (2017). Optimal reactive power scheduling using cuckoo search algorithm. International Journal of Electrical \& Computer Engineering, 7(5), 2349-2356.

Reddy, S.S., Bijwe, P.R., \& Abhyankar, A.R. (2015). Joint energy and spinning reserve market clearing incorporating wind power and load forecast uncertainties. IEEE Systems Journal, 9(1), 152-164.

Ren, K., \& Jihong, Q. (2014). A multiobjective interval programming model for wind-hydrothermal power system dispatching using 2-step optimization algorithm. The Scientific World Journal, 2014, Article ID 825216, 1-9. https://doi.org/10.1155/2014/825216.

Singh, S.N., Stergaard, J.Ø., \& Yadagiri, J. (2009). Application of advanced particle swarm optimization techniques to wind-thermal coordination. In 2009 15th International Conference on Intelligent System Applications to Power Systems (pp. 1-6). IEEE. Curitiba, Brazil.

Wood, A.J., Wollenberg, B. F., \& Sheblé, G.B. (2013). Power generation, operation, and control. John Wiley \& Sons. New York.

Zhang, Y., Le, J., Liao, X., Zheng, F., Liu, K., \& An, X. (2018). Multi-objective hydro-thermal-wind coordination scheduling integrated with large-scale electric vehicles using IMOPSO. Renewable Energy, 128, 91-107.

Zhou, W., Peng, Y., \& Sun, H. (2011). Optimal wind-thermal coordination dispatch based on risk reserve constraints. European Transactions on Electrical Power, 21(1), 740-756. 